\title{
Análisis dinámico de estabilidad de taludes por medio de elementos finitos
}

\section{Jaime Bojorque Iñeguez}

Facultad de Ingeniería, Universidad de Cuenca, Av. 12 de abril s/n, Cuenca, Ecuador.

Autor para correspondencia: jaime.bojorque@ucuenca.edu.ec

Fecha de recepción: 11 de julio 2016 - Fecha de aceptación: 22 de septiembre 2016

\section{RESUMEN}

En este documento se presentan los resultados de un análisis, parámetro a parámetro, que afecta el desplazamiento de un talud, cuando es sometida a cargas dinámicas. Se evalúa el efecto de la forma del registro dinámico a través de características como la frecuencia, amplitud y duración. Así también, se investigan las características del suelo considerando el peso unitario, módulo de Young, coeficiente de Poisson, cohesión, ángulo de fricción y amortiguamiento. El desplazamiento de la cresta de un talud puede indicar el desempeño de estabilidad, de ahí que el uso de técnicas numéricas como los elementos finitos contribuye a entender el efecto que podría tener un sismo (carga dinámica) sobre la generación de deformaciones. Métodos tradicionales con el pseudoestático o de Newmark no consideran las características del sismo. Para poder captar de mejor manera el efecto de un sismo se debe incorporar la frecuencia, amplitud y duración con el fin de ser capaces de hacer una estimación fiable de los desplazamientos, lo que se puede realizar a través de un análisis dinámico por elementos finitos.

Palabras clave: Estabilidad de taludes, análisis dinámico, elementos finitos.

\begin{abstract}
This manuscript presents the results of an analysis, parameter by parameter, that affect the displacement of a slope when subjected to dynamic loads. The effect of the shape of the dynamic record through parameters such as frequency, amplitude and duration is evaluated. Also, the soil characteristics are investigated considering the specific gravity, Young's modulus, Poisson's ratio, cohesion, friction angle and damping. The displacement of the crest of a slope can indicate the performance of stability, hence the use of numerical techniques such as finite element helps to understand the effect that an earthquake (dynamic load) could have on the generation of deformations. Traditional methods such as pseudostatic and Newmark do not consider the characteristics of the earthquake. To better capture the effects of an earthquake, parameters such as frequency, amplitude and duration should be considered as to make a reliable estimate of displacements, which can be done by a dynamic analysis using finite elements.
\end{abstract}

Keywords: Slope stability, dynamic analysis, finite element.

\section{INTRODUCCIÓN}

Durante un evento sísmico, la estabilidad en un talud puede disminuir de diferentes maneras (Hack et al., 2007): i) las ondas sísmicas generan tensiones adicionales al talud; ii) los desplazamientos del suelo generados por las ondas sísmicas pueden ser lo suficientemente grandes como para romper los enlaces entre las partículas del suelo, por lo tanto, la reducción de resistencia general del suelo; y iii) en suelos sueltos, el efecto de la carga cíclica puede dar lugar a la compactación. Si la presión de agua no puede ser eliminada lo suficientemente rápido, la presión de poro aumentará. Como resultado las tensiones efectivas se reducen hasta cero. Este fenómeno se conoce como licuefacción. 
En el análisis sísmico de taludes, dos tipos de metodologías suele ser empleadas (Day, 2001). La primera es el Análisis de Estabilidad por Inercia donde los materiales conservan su resistencia a la rotura durante el evento sísmico. Los métodos más comúnmente utilizados en el análisis de inercia son el enfoque pseudoestático (detalles del método puede encontrarse en Bojorque et al., 2007) y el método de Newmark (Newmark, 1965). El segundo tipo de evaluación sísmica es el Análisis de Estabilidad con Debilitamiento, donde los materiales experimentan una reducción significativa en la resistencia a la falla durante el terremoto. Los deslizamientos de flujo y la difusión lateral son dos casos de análisis de debilitamiento que implican la licuefacción del suelo.

La forma más común para representar un sismo en un lugar determinado es obtener la aceleración del suelo, velocidad o desplazamiento en función de tiempo. El resultado se denomina Registros de Aceleración del Sismo (strong motion records). Existen tres alternativas clásicas empleadas por los ingenieros para obtener los registros sísmicos de una región: (i) acelerogramas artificiales compatibles con un espectro determinado; (ii) acelerogramas sintéticos; y (iii) registros reales de acelerogramas (Bommer \& Acevedo, 2004). Con el incremento de registros de los datos reales de terremotos, el uso de estos últimos es aconsejable, evitando algunos inconvenientes presentes dentro de las otras alternativas. Los datos registrados sobre movimientos sísmicos están fácilmente disponibles para muchas partes del mundo (por ejemplo, Europa, Estados Unidos, Japón). Por otro lado, la falta de datos sismológicos disponibles en internet es marcado para otros países. La selección del registro de un movimiento telúrico (strong ground motion record), generalmente se lo debe realizar en base a tres criterios: la magnitud, la distancia desde la fuente (epicentro) hasta el sitio de estudio, y las características del terreno. Vale la pena mencionar que la magnitud afecta de una manera considerable la forma del espectro de respuesta, por lo tanto, la selección del registro.

En general, se han propuesto diferentes parámetros para tener en cuenta la severidad de un terremoto, por ejemplo, la magnitud y distancia de la fuente (Wilson \& Keefer, 1983). Otras características de los sismos como la aceleración máxima del suelo (peak ground acceleration PGA), la aceleración espectral (spectral acceleration), y la Intensidad Arias (Arias intensity), también han sido consideradas (Abrahamson \& Silva, 1997). La aceleración máxima (PGA) se define como el valor absoluto máximo de la aceleración sísmica registrado. Al considerar únicamente este parámetro, se deja de lado la influencia de la frecuencia y la duración del movimiento telúrico. La aceleración espectral (Sa) representa la aceleración máxima que un evento provocará en un oscilador lineal con un solo grado de libertad con un período natural y amortiguamiento determinado. La intensidad Arias (Ia) toma en consideración la amplitud, las frecuencias del registro, así como la duración del mismo y se expresa como: (Arias, 1970):

$$
\text { Ia }=\frac{\pi}{2 g} \int_{0}^{t}[a(t)]^{2} d t
$$

donde $g$ la aceleración de la gravedad, $a(t)$ es la aceleración del terreno en función del tiempo y $t$ es la duración total del evento sísmico.

Mientras que los métodos pseudoestáticos proporcionan un índice de estabilidad, los métodos basados en desplazamientos, además, ofrecen información sobre las deformaciones asociadas con la falla del talud. Los desplazamientos que se obtienen en este tipo de análisis son un indicador valioso para evaluar el desempeño del talud en un fenómeno sísmico. La evaluación de la estabilidad se puede realizar en términos del desplazamiento crítico, que se define como el desplazamiento inducido más allá del cual puede producirse un fallo general del talud. El valor real del desplazamiento crítico depende del comportamiento y la resistencia del suelo. En este contexto, Newmark (1965) introdujo un método que sirve de puente entre el enfoque pseudoestático simplista y el modelamiento sofisticado de elementos finitos (FEM). Con el avance del poder computacional, el análisis dinámico de la estabilidad de taludes se puede considerar para estudios de caso, explotando las ventajas de los métodos numéricos.

En este documento se presenta el análisis dinámico para la estabilidad de taludes identificando el efecto que tiene cada parámetro en el desplazamiento de la cresta del talud, este análisis se lo realiza empleando el método de elementos finitos. Se hace una descripción breve de los conceptos teóricos en el método de elementos finitos y se realiza un análisis paramétrico. 


\section{ANÁLISIS DINÁMICO POR ELEMENTOS FINITOS}

En el modelamiento de la respuesta dinámica de una estructura de suelo, el FEM considera la inercia del subsuelo y la influencia del tiempo de la carga. Adicionalmente, la amortiguación de material (damping) y/o la geometría pueden ser tomadas en cuenta. A diferencia del análisis de Newmark, en estos métodos se pueden obtener los desplazamientos de la masa deslizada en cualquier punto y a cualquier intervalo de tiempo. Por otra parte, los resultados en diferentes fases durante el terremoto proporcionan información sobre el comportamiento del talud.

Con el fin de reducir al mínimo la influencia de las condiciones de contorno, los límites laterales se deben seleccionar lo suficientemente lejos del talud. En un análisis dinámico, fronteras absorbentes se utilizan para disipar las ondas de tensión y evitan las reflexiones de las mismas al interior del cuerpo del suelo. En PLAXIS (PLAXIS, 2007), las condiciones de contorno absorbentes son modeladas por amortiguadores viscosos. El amortiguador asegura que un aumento en la tensión en el borde sea absorbido sin rebote. Los bordes absorbentes disipan las componentes normal y cortante mediante un amortiguador en base a la siguiente relación:

$$
\begin{gathered}
\sigma_{n}=-C_{1} \rho V_{p} \dot{u}_{x} \\
\tau=-C_{2} \rho V_{s} \dot{u}_{y}
\end{gathered}
$$

donde $C_{l}$ y $C_{2}$ son los coeficientes de relajación y $\rho$ es la densidad del suelo. La onda de velocidad primaria (o de presión) es $V_{p}$ y secundaria (o de corte) $V_{s}$ y se determina con:

$$
V_{p}=\sqrt{\frac{E(1-v)}{\rho(1+v)(1-2 v)}} \quad y \quad V_{s}=\sqrt{\frac{E}{2 \rho(1+v)}}
$$

con $\hat{u}_{x}$ y $\dot{u}_{y}$ siendo las velocidades horizontales y verticales, respectivamente. Fronteras absorbentes de este tipo funcionan bien cuando las ondas de presión sólo golpean al borde perpendicularmente (es decir $C_{l}=C_{2}=1$ ). En la presencia de ondas de corte que se propagan verticalmente, el efecto de estas fronteras es insuficiente, por ejemplo, durante un terremoto. De ahí que, PLAXIS recomienda utilizar $C_{l}=1$ y $C_{2}=0.25$, lo que resulta en la disipación adecuada de las ondas en la frontera. Este criterio es lo suficientemente preciso para la mayoría de las aplicaciones prácticas requeridas (PLAXIS, 2007).

El contorno inferior corresponde a la transición entre la roca madre y el suelo del talud. Los desplazamientos verticales no están permitidos a lo largo de esta frontera, mientras que la carga dinámica del terremoto (sismo) se aplica a través de desplazamientos horizontales previstos en la base. Estos desplazamientos se pueden obtener de acelerogramas o partir de las velocidades o desplazamientos.

El amortiguamiento es la pérdida de energía de los sistemas en vibración y puede ser producido por diferentes aspectos, tales como la pérdida de fricción y la no linealidad en la relación esfuerzodeformación del material. Todos los sistemas dinámicos contienen un cierto grado de amortiguación de la energía de vibración. Un ejemplo sencillo es un sistema masa-resorte amortiguado que se estira inicialmente. Cuando la fuerza de acción desaparece, la amortiguación por fricción del sistema reduce la amplitud de las oscilaciones hasta que se obtiene el equilibrio. El mismo efecto se observa en formaciones geológicas. La energía liberada por un terremoto se disipa debido a la pérdida de energía como consecuencia de la fricción interna entre las partículas del material.

Cabe señalar que, para un terremoto, la amortiguación geométrica puede ser significativa en la atenuación de la vibración. En el modelo de deformación plana que será usado en este análisis, sin embargo, la carga dinámica se aplica a lo largo de la base del modelo, lo que resulta en ondas de corte que se propagan hacia arriba. Por lo tanto, la amortiguación geométrica es insignificante, lo que lleva a un papel importante a la amortiguación del material con el fin de obtener resultados realistas. La amortiguación generalmente se modelada usando un término de amortiguamiento adicional que se añade a la ecuación de movimiento. Un parámetro de amortiguación utilizado comúnmente es el coeficiente de amortiguamiento $\xi$, que es igual a la relación de constante de amortiguamiento, $c$, para 
la constante de amortiguamiento crítico $c_{c}$, siendo ese último la amortiguación que trae al sistema al reposo en un tiempo mínimo (Kramer, 1996):

$$
\xi=\frac{c}{c_{c}}
$$

En el análisis dinámico de elementos finitos, la ecuación de movimiento bajo una aceleración $\left(\ddot{U}_{g}(t)\right)$ en la base que se puede escribir en forma matricial como:

$$
\mathbf{M} \ddot{U}+\mathbf{C} \dot{U}+\mathbf{K} U=-\mathbf{M I} \ddot{U}_{g}(t)
$$

donde $U, U$ y $\ddot{U}$ corresponde a las matrices de desplazamientos, velocidades y aceleraciones, respectivamente. $\mathbf{M}$ es la matriz de masa, $\mathbf{C}$ es la matriz de amortiguamiento, $\mathbf{K}$ es la matriz de rigidez, I es un vector unitario en las direcciones de análisis y cero en todas las demás posiciones, y $\ddot{U}_{g}(t)$ es la aceleración sísmica impuesta a lo largo del tiempo $t$. En PLAXIS, la matriz de amortiguación C utiliza los conceptos de amortiguación de Rayleigh, donde la matriz es proporcional a la masa y rigidez del sistema en función de:

$$
\mathbf{C}=\eta_{1} \mathbf{M}+\eta_{2} \mathbf{K}
$$

aquí $\eta_{1}$ y $\eta_{2}$ son los coeficientes de Rayleigh.

No se cuentan, sin embargo, con expresiones directas de $\eta_{1}$ y $\eta_{2}$. En lugar de ello, los valores se obtienen de manera indirecta a través del coeficiente de amortiguamiento $\xi$ a una frecuencia angular $\omega$ definida. Estos parámetros se relacionan con $n_{1}$ y $n_{2}$ por la siguiente expresión (Bathe \& Wilson, 1976):

$$
\eta_{1}+\eta_{2} \omega_{i}^{2}=2 \omega_{i} \xi_{i}
$$

Si se conocen dos relaciones de amortiguamiento a frecuencias determinadas, los parámetros $\eta_{1} \mathrm{y}$ $\eta_{2}$ se puede determinar. Los coeficientes de amortiguamiento de Rayleigh proporcionan una amortiguación aproximadamente constante en una gama limitada de frecuencias alrededor de la frecuencia angular $\omega_{\min }$. Por lo tanto, $\omega_{\min }$ se elige generalmente para mantenerse en el centro de la gama de frecuencias estimadas en la simulación numérica y $\xi_{\min }$ alrededor del factor de amortiguación que se puede determinar por medio de la prueba de columna resonante. Como resultado, los parámetros de Rayleigh se pueden determinar mediante:

$$
\begin{gathered}
\eta_{1}=\xi_{\text {min }} \omega_{\text {min }}=\xi_{\text {min }} 2 \pi f_{\text {min }} \\
\eta_{2}=\xi_{\text {min }} / \omega_{\text {min }}=\xi_{\text {min }} /\left(2 \pi f_{\text {min }}\right)
\end{gathered}
$$

donde $f_{\min }$ es la frecuencia central, igual a $\omega_{\min } / 2 \pi$.

En este estudio, la relación mínima de amortiguación $\xi_{\min }$ se supone igual a 5\% (salvo en el estudio paramétrico de la amortiguación). Vale la pena mencionar que 5\% es considerado adecuado para estudios de suelos (Bathe \& Wilson, 1976). El valor de $f_{\text {min }}$ se selecciona igual a la frecuencia del registro de aceleración. Para el análisis de sismos reales, $f_{\min }$ debe seleccionarse en base a la frecuencia característica del sitio.

\subsection{Efecto de sitio}

Los efectos de un sismo en dos lugares a distancias del epicentro idénticas pueden variar significativamente debido a las condiciones locales del suelo. Esto se debe al aumento de la intensidad sísmica debido a las características del terreno, que se denomina efectos del sitio (site effects) (Day, 2001). Características como la estratigrafía, la topografía y las propiedades mecánicas del suelo están involucradas al considerar los efectos del sitio. Al asumir que el suelo y roca base se extienden infinitamente y la respuesta del suelo es causada por la propagación vertical de las ondas sísmicas, la amplificación del sismo se puede determinar como: 


$$
|F(\omega)|=\left|\frac{u_{\max , s}}{u_{\max , b}}\right|=\frac{1}{\sqrt{\cos ^{2}\left(\frac{\omega H}{V_{s}}\right)+\left[\xi\left(\frac{\omega H}{V_{s}}\right)\right]^{2}}}
$$

donde $|F(\omega)|$ es la función de amplificación, $\omega$ es la frecuencia circular, $H$ el espesor del suelo, $V_{s}$ la velocidad de la onda de corte, y $\xi$ el coeficiente de amortiguamiento, $u_{\max , s}$ y $u_{\max , b}$ son los desplazamientos máximos horizontales en la superficie y base rocosa, respectivamente. Esta relación se obtiene considerando una capa uniforme isotrópica, lineal elástica y un suelo con amortiguación que se impone al movimiento sísmico.

La amplificación local máxima se encuentra cuando $\omega H / V_{s}=(2 n-1) \pi / 2$ (para $n=1,2, \ldots$ ). Las frecuencias que corresponden a estos máximos se llaman "frecuencias naturales". La primera frecuencia natural también se conoce como frecuencia fundamental y se determina con $n=1$ resultando:

$$
\omega_{1}=\frac{\pi V_{s}}{2 H}
$$

El período característico del sitio, que da una indicación del período de vibración al que se puede esperar que la amplificación sea máxima es:

$$
T_{s}=\frac{2 \pi}{\omega_{1}}=\frac{4 H}{V_{s}} \quad o \quad f_{s}=\frac{V_{s}}{4 H}
$$

En este modelo, la respuesta del suelo está predominantemente dada por las ondas de corte que se propagan verticalmente desde el lecho de roca subyacente. Para el análisis de taludes, la respuesta dinámica del talud se ve afectada por la masa del suelo con un espesor de $D H$, donde $D$ es el factor de profundidad. Considerando esto, el período característico del sitio de un talud se puede determinar como (Kramer \& Smith, 1997):

$$
T_{S}=\frac{4 D H}{V_{S}}
$$

\section{ESTUDIO PARAMÉTRICO PARA LA EVALUACIÓN DINÁMICA}

En esta sección se evalúa el efecto de los parámetros más importantes en el análisis dinámico de estabilidad de taludes. Un análisis paramétrico se realiza para detectar el efecto de la frecuencia, la aceleración máxima, la duración, los parámetros geométricos y las propiedades del suelo en los desplazamientos totales. Se emplea el programa de elementos finitos PLAXIS cuya teoría se ha indicado en la sección anterior.

La sensibilidad de cada parámetro, en el desempeño del talud, se evalúa mediante el desplazamiento vertical de la parte superior. Con la finalidad de caracterizar de mejor manera cada parámetro, en el presente estudio, se aplican registros armónicos para analizar el efecto de la frecuencia, aceleración máxima y duración del evento. El talud a analizar corresponde a un talud homogéneo con una inclinación $\alpha$, altura $H$ y factor de profundidad $D$. En la Figura 1 se presenta la representación esquemática del modelo del talud a emplearse.

La aceleración se aplica en la parte inferior del modelo, caracterizada por una frecuencia $(f)$, aceleración máxima $(P G A)$ y duración $(T d)$. Para estudiar la influencia del registro dinámico, se suponen las siguientes propiedades del suelo: peso unitario $\gamma=17 \mathrm{kN} / \mathrm{m}^{3}$, Módulo Elástico de Young $E=30 \mathrm{MPa}$, Coeficiente de Poisson $v=0.3$, cohesión $c=8 \mathrm{kPa}$, ángulo de fricción $\phi=20^{\circ}$. El coeficiente de amortiguamiento, $\xi$ se considera que es constante e igual a $5 \%$. Para evaluar el efecto de las propiedades del suelo (es decir, $\gamma, E, v, c, \phi$ y $\xi$ ), cada parámetro se varia de forma sistemática. El suelo se modela como un material perfectamente elasto-plástico, obedeciendo al criterio de falla de MohrCoulomb. 


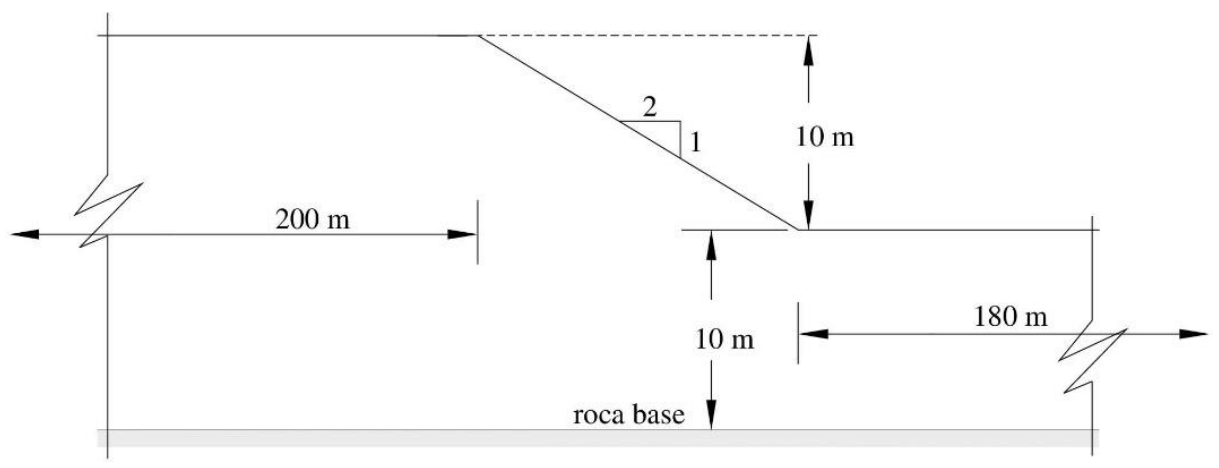

Figura 1. Modelo empleado en el análisis paramétrico.

La influencia de las características del movimiento sísmico en el desplazamiento del talud es analizada empleando un registro de ingreso de aceleración armónica. Esta aceleración se puede caracterizar por la frecuencia $f(\mathrm{~Hz})$, la aceleración máxima $\left(P G A\right.$, en g ó $\left.\mathrm{m} / \mathrm{s}^{2}\right)$ y la duración $T d$ (segundos). El talud tiene una inclinación $\alpha=26.6^{\circ}(2 \mathrm{H}: 1 \mathrm{~V}), H=10$ m y $D=2$.

Es importante mencionar que, para casos de estudio con frecuencias bajas, el modelo falla en la base y la superficie del modelo se levantada hacia arriba. Por lo tanto, la parte superior del talud no sólo se mueve hacia abajo, sino también hacia arriba debido al fracaso en la base. En consecuencia, los desplazamientos verticales son alterados (Timmermans, 2008). Con la finalidad de superar este problema, se han estudiado diferentes modelos. El modelo seleccionado, para el estudio paramétrico, corresponde a un modelo que incluye un lecho de roca rígida.

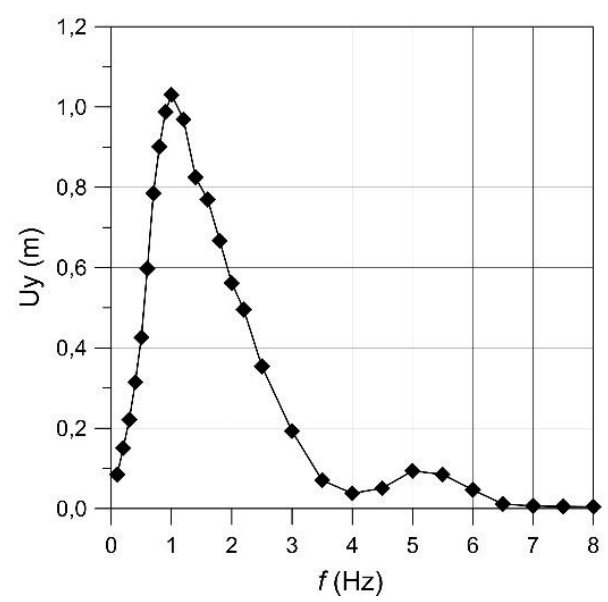

Figura 2. Desplazamientos verticales totales en la cresta del talud para frecuencias desde $f=0.1-8 \mathrm{~Hz}$.

\subsection{Efecto de la frecuencia $(f)$}

La influencia de la frecuencia $f$ es analizada empleando un rango desde 0.1 a $8 \mathrm{~Hz}$. La aceleración máxima se mantiene constante equivalente $2,5 \mathrm{~m} / \mathrm{s}^{2}$ equivalente a $0.255 \mathrm{~g}$ y la duración se asigna un valor de $10 \mathrm{~s}$, seguido de $4 \mathrm{~s}$ de movimiento libre. Para cada frecuencia, el desplazamiento vertical máximo $\left(u_{y}\right)$ en la cresta del talud es determinado. En la Figura 2 se muestran los resultados del desplazamiento en mención en función de la frecuencia del registro dinámico.

Los mayores desplazamientos se obtienen para las frecuencias cercas a $1 \mathrm{~Hz}$, correspondiente a la frecuencia fundamental del sitio $f_{s}=1,02 \mathrm{~Hz}$. La amplificación del registro de entrada es máxima para las frecuencias cercanas a la frecuencia fundamental. Se puede apreciar en la Figura 2 la presencia de un segundo pico que se presenta en $f=5 \mathrm{~Hz}$, lo cual está en concordancia con la tercera frecuencia natural del sitio, $f_{3}=5 f_{s}=5.1 \mathrm{~Hz}$. Este máximo local se produce por la amplificación a través del suelo. Sin embargo, para la segunda frecuencia natural $f_{2}=3 f_{s}=3.06 \mathrm{~Hz}$, esta amplificación no puede ser observada debido al tamaño del primer pico. 
El fenómeno más importante es el efecto de la resonancia cuando la frecuencia del registro está cercana a la frecuencia fundamental. Cuando $f$ se acerca $f_{s}$, se genera una importante amplificación de la aceleración a lo largo del suelo, lo que lleva a un aumento de los esfuerzos resultando en mayores deformaciones del talud y por ende mayores desplazamientos de la cresta. Del desplazamiento vertical en función del tiempo, para las frecuencias $f=0.5 \mathrm{y} 1 \mathrm{~Hz}$, se observa que después de $14 \mathrm{~s}$ los desplazamientos resultantes son 0.44 y $1.03 \mathrm{~m}$, respectivamente. Para $f=1 \mathrm{~Hz}$, los desplazamientos ascendentes son insignificantes, resultando en un desplazamiento final superior, mientras que para $f=$ $0.5 \mathrm{~Hz}$ la oscilación en los desplazamientos de arriba-abajo se produce en cada ciclo, lo que resulta en desplazamientos totales más bajos.

La curva de la aceleración en la cresta y base del modelo para $f=0.5$ y $1 \mathrm{~Hz}$ se muestra en la Figura 3. En esta figura, la aceleración horizontal $\left(a_{x, t}\right)$ y vertical $\left(a_{y, t}\right)$ de la cresta y aceleración horizontal de la base $\left(a_{x, b}\right)$ están graficadas. Se puede apreciar claramente la amplificación del registro a través del suelo, especialmente en los picos positivos. Las aceleraciones negativas son amplificadas en menor medida debido a que la parte superior se ve más limitada en dirección del talud, mientras que las aceleraciones positivas, debido a la presencia de una superficie libre, conducen a una mayor amplificación. La masa deslizante inicia a moverse en dirección positiva tan pronto como la superficie de falla se ha desarrollado.

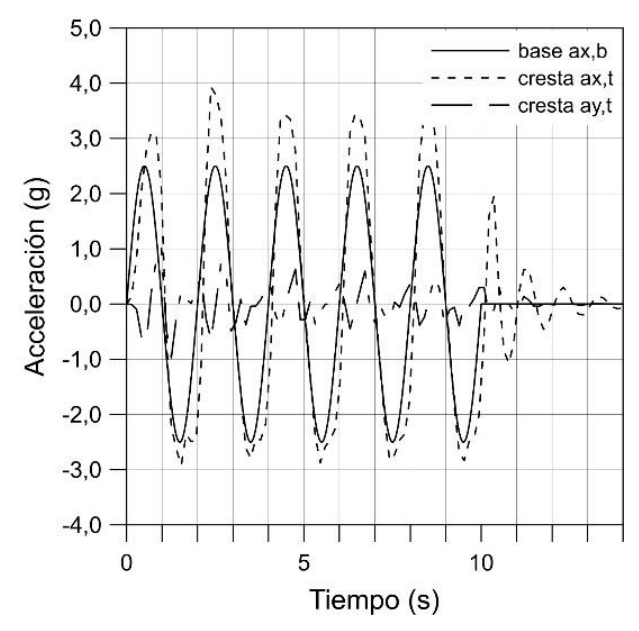

(a)

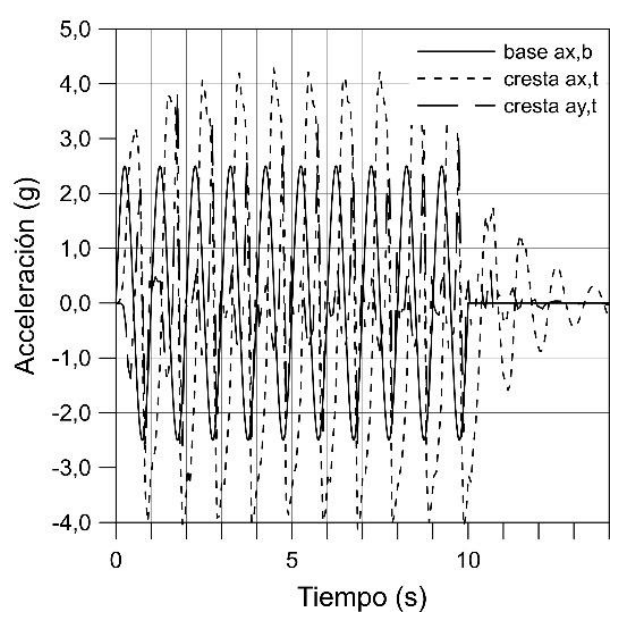

(b)

Figura 3. Aceleración horizontal en la cresta $a_{x, t}$ (línea discontinua fina), vertical $a_{y, t}$ (línea discontinua gruesa) y aceleración horizontal en la base $a_{x, b}$ (línea sólida) para: (a) $f=0.5 \mathrm{~Hz}$ y (b) $f=1$ $\mathrm{Hz}$.

Para $f=1 \mathrm{~Hz}$, el desplazamiento vertical $\left(u_{y}=1,03 \mathrm{~m}\right)$ resultando dos veces mayor que para $f=0.5$ $\mathrm{Hz}\left(u_{y}=0.42 \mathrm{~m}\right)$. Las aceleraciones horizontales en la cresta son $60 \%$ superiores a las de la base. Los picos de la aceleración horizontal de la cresta muestran una vez más puntos de corte, pero esta vez para los valores positivos, así como para las aceleraciones negativas. A diferencia que para el caso en que $f=0.5 \mathrm{~Hz}$, el desplazamiento se produce dos veces durante cada período de vibración, debido al aumento de la amplificación de las aceleraciones. Esto también se observa en la aceleración vertical de la parte superior $\left(a_{y, t}\right)$, que muestra dos picos negativos durante cada ciclo a $1 \mathrm{~s}$ (Figura $3 \mathrm{~b}$ ). Como resultado, se genera un desplazamiento de la cresta hacia abajo, como lo ilustra el esquema de desplazamientos en la Figura 3a. Este efecto también ha sido observado por Wartman et al. (2003), teniendo en cuenta el desplazamiento inducido por vibraciones sobre un plano inclinado.

\subsection{Efecto de la máxima aceleración (PGA)}

Para el movimiento armónico, la $P G A$ puede ser expresada por la amplitud de la función sinusoidal. En este estudio, $P G A$ oscila entre $0.1 \mathrm{a} 5 \mathrm{~m} / \mathrm{s}^{2}$. El desplazamiento vertical de la cresta frente a la máxima aceleración se muestra en la Figura 4. Como es de esperarse, al aumentar PGA, los desplazamientos permanentes también aumentan. Para frecuencias cercanas a la frecuencia fundamental del sitio los 
desplazamientos totales son considerables. Para frecuencias $f\left\langle\left\langle f_{s}\right.\right.$ ó $\left.f \gg\right\rangle f_{s}$, los desplazamientos son menos importantes.



Figura 4. Desplazamientos verticales totales en la cresta versus aceleración máxima $P G A$ para varias frecuencias $f$.

Para valores bajos de $P G A$, el desplazamiento aumenta con el aumento de la aceleración, mientras que, para valores mayores la curva se hace menos empinada y muestra una tendencia más lineal. Hay que tener en cuenta que la aceleración máxima $(P G A)$ no es el único factor determinante, aquí, la frecuencia, también, tiene una gran influencia en la forma de las curvas. Para frecuencias cercanas a la frecuencia fundamental, se producen grandes desplazamientos, incluso para aceleraciones bajas. Para $f=f_{s}=1 \mathrm{~Hz}$ y $P G A=0.50 \mathrm{~m} / \mathrm{s}^{2}$ (valor inferior al de la aceleración crítica de $1.42 \mathrm{~m} / \mathrm{s}^{2}$ ), el desplazamiento es de $1.8 \mathrm{~m}$ hacia abajo. Esto es nuevamente el resultado de la amplificación de las aceleraciones a lo largo del suelo. En consecuencia, las aceleraciones amplificadas en el suelo sobrepasan la aceleración crítica y se genera una superficie deslizante. Cuando la frecuencia del registro está muy lejos de la frecuencia fundamental del sitio, los desplazamientos son insignificantes, por ejemplo, para $f=8 \mathrm{~Hz}, \mathrm{y}$ $P G A=0.50 \mathrm{~m} / \mathrm{s}^{2}$ se tiene como resultado $u_{y}=0.026 \mathrm{~m}$.

\subsection{Efecto de la duración del evento $(T d)$}

Para los registros armónicos, la duración es igual a la duración del movimiento sinusoidal. Para evaluar la influencia de la duración, este parámetro se varió entre 0.5 a $40 \mathrm{~s}$, mientras que la frecuencia se mantuvo constante en 1.25 y $2.5 \mathrm{~Hz}$, la aceleración máxima fue de 0.5 y $4 \mathrm{~m} / \mathrm{s}^{2}$. Los desplazamientos verticales en la cresta de este análisis se muestran en la Figura 5. Se observa que el aumento de la duración provoca el aumento de los desplazamientos en una tendencia perfectamente lineal, con coeficientes de regresión próximo a la unidad. Para mayor aceleración máxima, o cuando $f$ está cerca de la frecuencia fundamental, los desplazamientos son más significativos.

\subsection{Efecto de las propiedades del suelo}

En esta sección, se analiza el efecto de las propiedades del suelo con relación a los desplazamientos totales. Las propiedades del suelo que serán consideradas son: peso unitario $(\gamma)$, el módulo de Young $(E)$, el coeficiente de Poisson $(v)$, la cohesión $(c)$, el ángulo de fricción $(\phi)$, y la amortiguación del material $(\xi)$. Al igual que en el análisis anterior, el talud tiene una altura de $10 \mathrm{~m}$, con una inclinación de $2 \mathrm{H}: 1 \mathrm{~V}$ y una capa inferior con espesor igual a la altura del talud $(D=2)$. Los parámetros del suelo considerados son: $\gamma=17 \mathrm{kN} / \mathrm{m}^{3}, E=30 \mathrm{MPa}, v=0.3, \xi=5 \%, c=8 \mathrm{kPa}$, y $\phi=20^{\circ}$. Los registros dinámicos tienen una amplitud $P G A=1.5 \mathrm{~m} / \mathrm{s}^{2}$, mientras que la duración del movimiento $T d$ es igual a $10 \mathrm{~s}$, después de lo cual se deja sin carga por $4 \mathrm{~s}$. La frecuencia de ingreso $f$ se varía entre $0.1 \mathrm{a} 8 \mathrm{~Hz}$, con el fin de estudiar la interacción entre los parámetros del suelo y la frecuencia. Dependiendo de qué parámetro se analiza, su valor se cambia sistemáticamente y su cambio se mencionará en cada sección. 


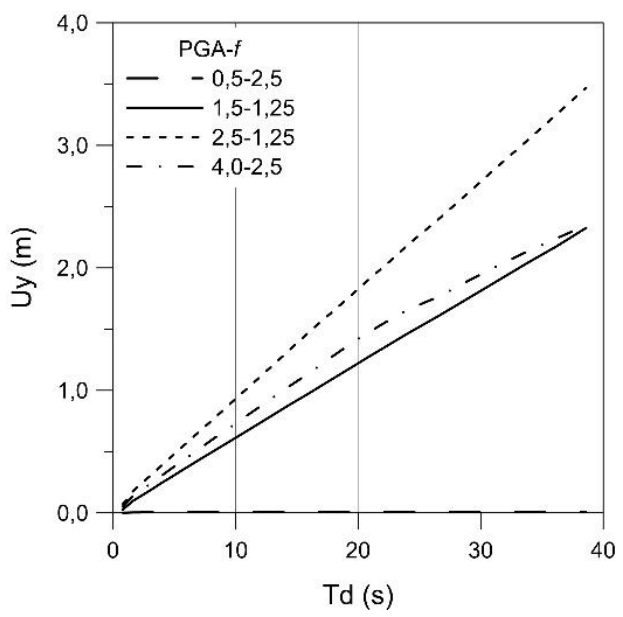

Figura 5. Desplazamiento vertical en la cresta versus la duración $T d$ para valores de $P G A=1.5$ y 2.5 $\mathrm{m} / \mathrm{s}^{2}$ y $f=1.25$ y $2.5 \mathrm{~Hz}$.

\subsection{Efecto del peso unitario $(\gamma)$}

El efecto del peso unitario $(\gamma)$ es analizado cambiando su valor de 10 a $22 \mathrm{kN} / \mathrm{m}^{3}$. Se conoce que un aumento en el peso unitario reduce la estabilidad de los taludes ya que el aumento de peso del suelo también aumenta la fuerza actuante. En la Tabla 1 se presenta los resultados de un análisis de taludes estático y pseudoestático para diferentes pesos unitarios.

Tabla 1. Factor de seguridad, coeficiente sísmico y frecuencias características del sitio para diferentes pesos unitarios.

\begin{tabular}{lcccc}
\hline & \multicolumn{4}{c}{ Peso unitario $(\gamma)\left(\mathrm{kN} / \mathrm{m}^{3}\right)$} \\
\hline Factor de seguridad & 10 & 14 & 18 & 22 \\
Coeficiente sísmico & 1.65 & 1.45 & 1.34 & 1.27 \\
Frecuencia del sitio & 1.33 & 1.12 & 0.99 & 0.90 \\
\hline
\end{tabular}

Para el análisis dinámico, al incrementar el peso unitario se puede esperar la reducción de la estabilidad del talud dando lugar a deformaciones significativas. El cambio en $\gamma$ también produce un cambio en la velocidad de onda de corte $V_{s}$, por lo tanto, se afecta la amplificación de las ondas a través del suelo, lo que resulta en un cambio en la característica de frecuencia del sitio (Tabla 1).

La influencia del peso unitario en el desplazamiento vertical se muestra en la Figura 6. Un cambio en $\gamma$ resulta en un cambio del desplazamiento máximo, por lo tanto, el suelo más denso es más vulnerable a los sismos con contenido de frecuencias cercanos a $f_{s}$. El desplazamiento máximo vertical de la cresta (picos a $f=f_{s}$ ) aumenta con la densidad del suelo. Este comportamiento tiene una tendencia lineal entre el peso unitario del suelo y el desplazamiento máximo de la cresta $\left(u_{y, \max }\right)$.

\section{6. $\quad$ Efecto del módulo de Young (E)}

En este análisis, para el módulo de Young se asignan valores de 10, 30, 70, 100 y 300 MPa para ver su influencia. Para los análisis de estabilidad de los taludes estáticos, sólo un análisis es necesario ya que la estabilidad es independiente de la rigidez del suelo. El factor de seguridad y coeficiente sísmico críticos para este caso son 1.36 y $0.14 \mathrm{~g}$, respectivamente. En la Figura 7 se muestra el desplazamiento vertical en la cresta en función de la frecuencia para los cinco módulos de Young analizados. 


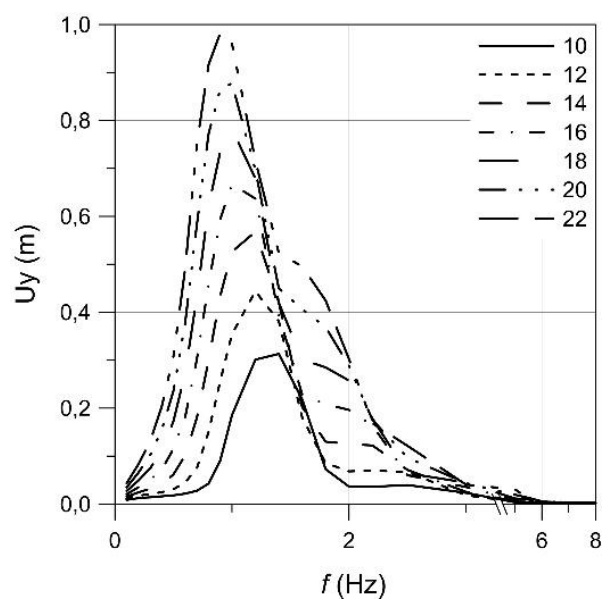

Figura 6. Efecto del peso unitario en los desplazamientos verticales de la cresta: (a) $u_{y}$ vs $f$ para diversos $\gamma\left(10\right.$ a $\left.22 \mathrm{kN} / \mathrm{m}^{3}\right)$ y (b) $u_{y, \max } \mathrm{vs} \gamma$ para $f=f_{s}$.

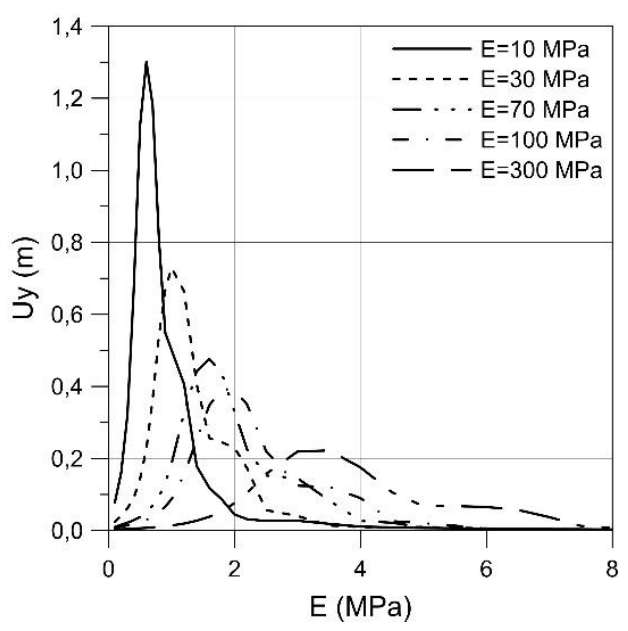

Figura 7. Efecto del módulo de Young en los desplazamientos verticales en la cresta.

Los resultados muestran un desplazamiento del pico máximo de resonancia para frecuencias más altas. Este comportamiento se debe al cambio en la frecuencia del sitio. Al aumentar los valores de rigidez, la onda de velocidad de corte aumenta, por lo tanto, la frecuencia característica del sitio varía. Todas las curvas también muestran un pico asimétrico, como consecuencia de la resonancia en la capa de base para $f=2 f_{s}$. Al aumentar la rigidez del suelo, incrementando así $f_{s}$, el desplazamiento máximo de la cresta disminuye. Esto no es el resultado de una disminución en la amplificación de las frecuencias, ya que el factor de amplificación de la frecuencia fundamental es independiente de la rigidez del suelo, donde:

$$
\begin{gathered}
\omega=\omega_{1}=\frac{\pi v_{s}}{2 H} \\
|F(\omega)|=\left|\frac{u_{\max , s}}{u_{\max , b}}\right|=\frac{1}{\sqrt{1+[\xi(\pi / 2)]^{2}}}
\end{gathered}
$$

Sin embargo, para frecuencias más altas, la amplitud del desplazamiento que se aplica en la roca base es menor que para las frecuencias más bajas. Como resultado, los desplazamientos totales de la cresta son más pequeños para las frecuencias más altas. 


\subsection{Efecto del coeficiente de Poisson (v)}

El coeficiente de Poisson para este análisis varía entre 0.20 a 0.45 con incrementos de 0.01 . La influencia de $v$ en el desplazamiento vertical en la cresta se muestra en la Figura 8.

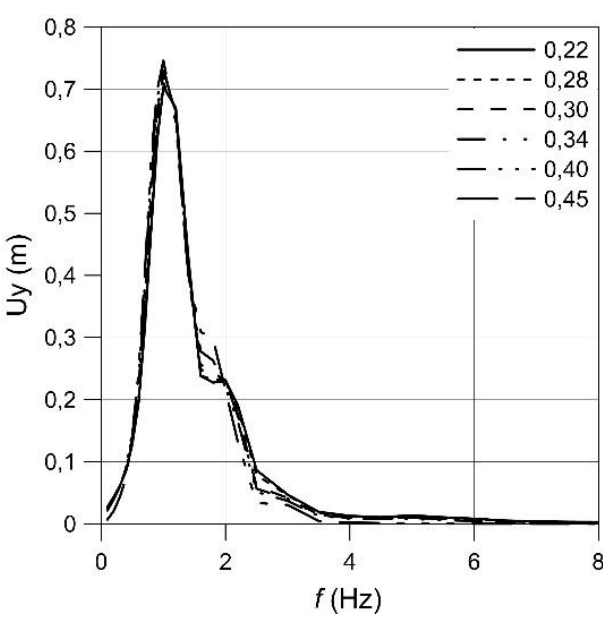

(a)

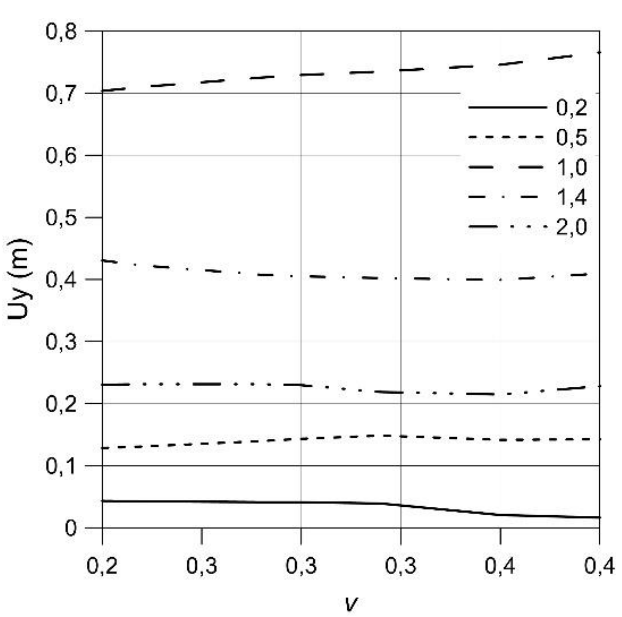

(b)

Figura 8. Efecto del coeficiente de Poisson $(v)$ en los desplazamientos verticales en la cresta: (a) $f$ para varios $v(0.20$ a 0.45$)$ y (b) $v$ para varias $f$.

A diferencia del módulo de Young, este parámetro tiene un efecto insignificante sobre los resultados. Mientras que la velocidad de onda de corte $V s$ y la frecuencia característica del sitio depende tanto de $E$ y $v$, este último tiene poco efecto sobre su valor. Por ejemplo, cuando $v=0.20, V s=84.9$ $\mathrm{m} / \mathrm{s}$ y $f_{s}=1.06 \mathrm{~Hz}$, mientras que cuando $v=0.45$ se obtiene $f_{s}=0.97 \mathrm{~Hz}$. Se debe considerar que, a pesar de que $v$ es dos veces el valor, las frecuencias del sitio son casi las mismas. La Figura $8 \mathrm{~b}$ presenta el desplazamiento vertical máximo para el coeficiente de Poisson versus la frecuencia. Cambiando $v$ los desplazamientos verticales máximos en la cresta, no varían considerando la misma frecuencia.

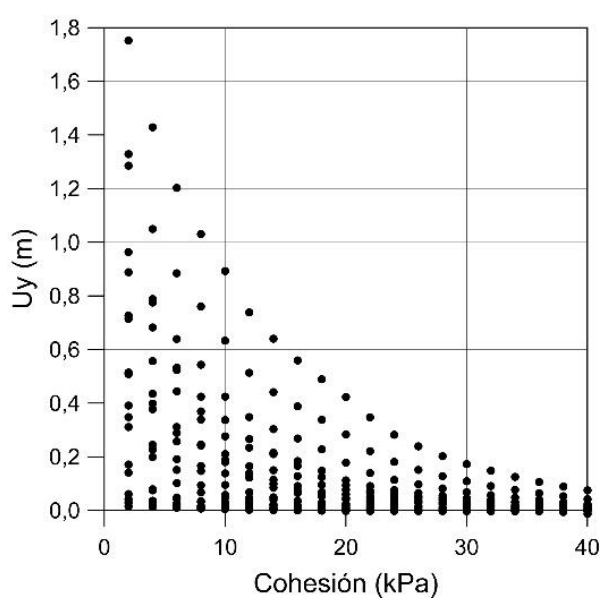

(a)

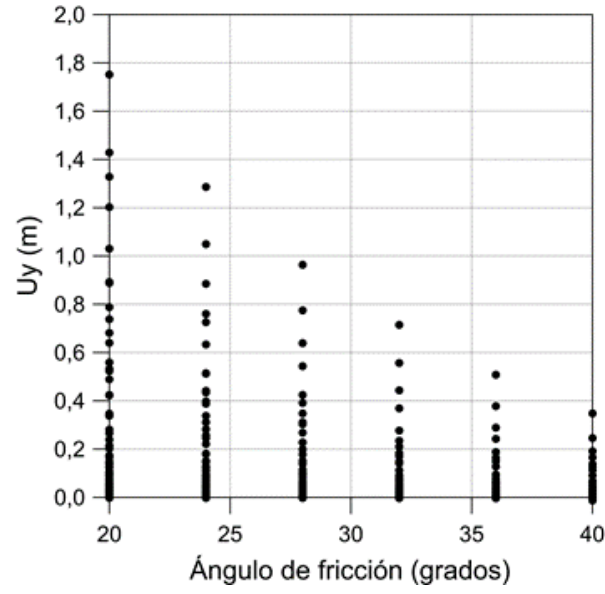

(b)

Figura 9. Efecto de los parámetros de resistencia en los desplazamientos verticales en la cresta: a) cohesión $(2$ a $40 \mathrm{kPa})$ y b) ángulo de fricción $\left(20\right.$ a $\left.40^{\circ}\right)$.

\subsection{Efecto de la resistencia del material, cohesión (c) y ángulo de fricción (phi)}

Los parámetros empleados para este análisis fueron, duración $T d=10 \mathrm{~s}$ y la amplitud $P G A=2.5 \mathrm{~m} / \mathrm{s}^{2}$. Tres diferentes frecuencias de entrada se seleccionaron: $f=0.5,1$ y $2.5 \mathrm{~Hz}$. La cohesión se varió de 2 a $40 \mathrm{kPa}$ y el ángulo de fricción de $20 \mathrm{a} 40^{\circ}$. La influencia de la cohesión y el ángulo de fricción sobre los desplazamientos verticales se muestran en la Figura 9a y b, respectivamente. La cohesión y ángulo 
de fricción afectan a los desplazamientos totales de diferente forma. Sin embargo, en ambos casos, a medida que la cohesión o el ángulo de fricción aumentan, los desplazamientos verticales en la cresta disminuyen.

El factor de seguridad o coeficiente sísmico representan un índice de estabilidad, que abarca los parámetros de resistencia $c$ y $\phi$, estos parámetros se evalúan según los desplazamientos verticales en la cresta. Se determinó que existe una relación de $u_{y}$ y el factor de seguridad $(F o S)$ y coeficiente sísmico $(k c)$. Realizando un ajuste del desplazamiento versus $c, \phi, F o S, k c$ se determinan valores de correlación de $51,38,63$ y $70 \%$, respectivamente.



Un valor comúnmente empleado para el amortiguamiento en el suelo es 5\%, aunque valores diferentes se pueden utilizar en casos especiales. El modelo del suelo es el mismo que los casos antes mencionados. Las aceleraciones armónicas que se utilizan tienen un $P G A=1.5 \mathrm{~m} / \mathrm{s}^{2}, T d=10 \mathrm{~s}$ y $f=0.1$ a $8 \mathrm{~Hz}$. Se emplearon seis relaciones de amortiguación $(\xi)$ de: 2, 4, 5, 6, 8 y 10\%. En la Figura 10 se presentan los desplazamientos en la cresta en función de la frecuencia para diferentes valores de amortiguación. Debido al concepto del $\xi$, no es una sorpresa que cuando $\xi$ disminuye, las aceleraciones serán menos disipadas (amortiguadas), con esfuerzos más altos como consecuencia. El resultado es que los desplazamientos en la cresta son más altos cuando se reduce la amortiguación.



Figura 10. Desplazamientos verticales frente a la frecuencia para varias relaciones de amortiguamiento ( $\xi$ desde 2 a 10$)$.

\section{DISCUSIÓN Y CONCLUSIONES}

En la presente investigación se realizó un estudio paramétrico con la finalidad de evaluar los efectos de las características del registro dinámico (a saber, frecuencia, amplitud y duración) y las características del suelo (peso unitario, módulo de Young, coeficiente de Poisson, cohesión, ángulo de fricción y amortiguamiento) sobre la estabilidad dinámica de los taludes. Aquí, se evalúa la estabilidad del talud a través de los desplazamientos verticales en la cresta.

Del análisis de las características del registro dinámico, se observa que la amplitud y la duración presentan una tendencia lineal con los desplazamientos totales, con la existencia de un umbral que depende de la frecuencia. Para la frecuencia, el efecto de resonancia $\left(f=f_{s}\right)$ tiene un efecto importante en los desplazamientos totales. Para frecuencias cercas a la frecuencia característica del sitio, se produce una considerable amplificación de movimientos en todo el suelo, aumentado las deformaciones totales. Este efecto se debe considerar en el análisis de registros sísmicos reales que consisten en una amplia gama de frecuencias. En este sentido, dos eventos sísmicos con características similares ( $P G A$ y 
duración $T d$ ) pueden tener un potencial destructivo muy diferente, por ejemplo, si la frecuencia del terremoto está cercana a la frecuencia natural del sitio causaría la inestabilidad. Para frecuencias inferiores a la frecuencia fundamental del sitio, las deformaciones totales tienden a disminuir, pero aún pueden ser significativas para valores altos de $P G A$. La misma tendencia decreciente se observa para frecuencias muy por encima de la frecuencia fundamental. Este análisis revela que ningún parámetro puede capturar todas las características de movimiento sísmico. Sin embargo, para poder captar de mejor manera el efecto de un sismo se debe incorporar los efectos como la frecuencia, amplitud y duración con el fin de ser capaces de hacer una estimación fiable de los desplazamientos.

Para los parámetros del suelo, se observa que una disminución en la resistencia (cohesión y ángulo de fricción) reduce la estabilidad del talud y por lo tanto provoca un aumento en los desplazamientos totales. Otros parámetros del suelo pueden, sin embargo, cambiar la frecuencia característica del sitio, cambiando el pico de los desplazamientos máximos a otra frecuencia, este es el caso de la rigidez, expresada a través del módulo de Young. Parámetros como el peso unitario causan una reducción en la estabilidad estática, así como un cambio en la característica de frecuencia sitio y cambios en los desplazamientos totales. La influencia del coeficiente de Poisson es insignificante, pues no influye en mayor medida en el análisis dinámico de la estabilidad del talud.

\section{AGRADECIMIENTOS}

Las corridas numéricas empleadas en el presente documento se las desarrolló durante el periodo de estudios doctorales en la Katholieke Universiteit Leuven, Bélgica, en el trabajo Bojorque Iñeguez, J.A., 2009. "Static and Dynamic Finite Element Analysis of Slope Stability".

\section{BIBLIOGRAFÍA}

Abrahamson, N.A., W.J. Silva, 1997. Empirical response spectral attenuation relations for shallow crustal earthquakes. Seismological Research Letters, 68(1), 94-127.

Arias, A., 1970. A measure of earthquake intensity. In: Hansen, J. (Ed.) Seismic Design for Nuclear Power Plants, 438-483. Cambridge, Massachusetts Institute of Technology Press.

Bathe, K.J., E.L. Wilson, 1976. Numerical methods in finite element analysis. Englewood Cliffs, NJ:: Prentice Hall Publishing.

Bojorque J., G. De Roeck, J. Maertens, 2007. Determination of the critical seismic acceleration coefficient in slope stability analysis using finite element methods. Int. Congress on Development, Environment and Natural Resources: Multi-level and Multi-scale Sustainability, Cochabamba, Bolivia.

Bommer, J., A. Acevedo, 2004. The use of real earthquake accelerograms as input to dynamic analysis. Journal Earthquake Engineering, 8(1), 43-91.

Day, R.W., 2001. Geotechnical earthquake engineering handbook ( $1^{\text {st }}$ ed.). New York City, NY: McGraw-Hill Publ. Comp.

Hack, R., D. Alkema, G. Kruse, N. Leenders, L. Luzi, 2007. Influence of earthquakes on the stability of slopes. Engineering Geology, 91(1), 4-15.

Kramer, S.L., 1996. Geotechnical earthquake engineering. Upper Saddle River, NJ: Prentice Hall Publishing.

Kramer, S.L., M.W. Smith, 1997. Modified Newmark model for seismic displacements of compliant slopes. Journal of Geotechnical and Geoenvironmental Engineering, 123(7), 635-644.

Newmark, N.M., 1965. Effects of earthquakes on dams and embankments. Géotechnique, 15(2), 139-160.

Plaxis, 2007. 2D-Version 8, finite element code for soil and rock analyses. Delft, The Netherlands. 
Timmermans, P., 2008. Seismic slope stability analysis. Master's thesis, Katholieke Universiteit Leuven, Belgium.

Wartman, J., J. Bray, R. Seed, 2003. Inclined plane studies of the Newmark sliding block procedure. Journal of Geotechnical and Geoenvironmental Engineering, 129(8), 673-684.

Wilson, R.C., D.K. Keefer, 1983. Dynamic analysis of a slope failure from the 6 August 1979 Coyote Lake, California Earthquake. Bulletin of the Seismological Society of America, 73, 863-877. 